\title{
The Growing Digital Divide and its Negative Impacts on NASA's Future Workforce
}

Corresponding Author: Moses P. Milazzo, Other Orb LLC, (513) 620-4314, moses@otherorb.net, (He/Him)

\section{Co-Authors:}

Christina Richey, Jet Propulsion Laboratory, California Institute of Technology (They/Them) Jennifer Piatek, Central Connecticut State University (She/Her)

Alicia Vaughan, Apogee Engineering LLC (She/Her)

Aparna Venkatesan, University of San Francisco (She/Her)

\section{Endorsements (Endorsement form; Active list of endorsements)}

Abbie Grace, University of Tasmania (she/her); Alfred S. McEwen, University of Arizona (he/him); Caela Barry, ADNET Systems, NASA Goddard Space Flight Center (she/her); Carol Paty, University of Oregon (she/her); Claire Newman, Aeolis Research (she/her); Daniel Glavin, NASA Goddard Space Flight Center (he/his); Daniella Scalice, NASA Ames Research Center (she/her); Devanshu Jha, MVJ College of Engineering, Bangalore, India; Evan L. Sneed, Pennsylvania State University (he/him or they/them); Gregory Schmidt, NASA Ames Research Center, Director, SSERVI (he/him); Gwen Hanley, University of California Berkeley (she/her); Henry G. Roe, Gemini Observatory, NSF's NOIRLab (he/him); Jacob Richardson, University of Maryland College Park (he/him); James Roberts, Johns Hopkins Applied Physics Laboratory; Jamie L. Molaro, Planetary Science Institute (she/her); Jani Radebaugh, MAPSIT chair (she/her); Jeffrey M. Moore, NASA ARC; Jennifer Hanley, Lowell Observatory (she/her); Jessica L. Noviello, Arizona State University (she/her); Kat Gardner-Vandy, Oklahoma State University; Kelsi N. Singer, Southwest Research Institute (she/her); Kristina Gibbs, NASA Ames Research Center; Kunio M. Sayanagi, Hampton University (he/him); Linda Spilker, JPL (she/her); M. Elise Rumpf, USGS Astrogeology Science Center; Maggie McAdam, NASA Ames Research Center; Margaret E. Landis, University of Colorado, Boulder/LASP (she/her); Martha Gilmore, Wesleyan University; Nicole Whelley, University of Maryland - College Park / NASA GSFC (she/her); Parvathy Prem, Johns Hopkins Applied Physics Laboratory; Romy D. Hanna, University of Texas at Austin (she/her); Ryan Watkins, Planetary Science Institute; Sanlyn Buxner, Planetary Science Institute; Serina Diniega, Jet Propulsion Laboratory, California Institute of Technology (she/her); Shawn Brueshaber, Jet Propulsion Laboratory, California Institute of Technology; Shoshana B. Cole, Space Science Institute (she/her); Steven Vance, Jet Propulsion Laboratory, California Institute of Technology (he/him); Timothy D. Glotch, Stony Brook University (he/him); Zibi Turtle, Johns Hopkins Applied Physics Laboratory (she/her) 
Introduction: There have been many white papers submitted to this Decadal Survey that have focused on representation of various people in the planetary science and astrobiology communities (e.g., references 1-10). The global pandemic, and especially its impact on education in the United States, has exposed that the digital inequality divide has been growing and its growth is accelerating ${ }^{1}$. This is an issue that will contribute to NASA's difficulty in meeting the recommendations in some of the associated white papers regarding the State of the Profession: Diversity, Equity, Inclusion, and Accessibility (DEIA). The negative impact will be felt especially by potential future members of the planetary sciences and astrobiology community, many of whom are just now entering high school or beginning their undergraduate education. Here we discuss the current state of the digital divide, its accelerating growth as exposed by the pandemic ${ }^{2}$, its impact on today's middle school, high school, and undergraduate students, its unequal impact on various communities in the US, and the implications for the future workforce. This white paper is submitted as part of a collaborative effort organized by the Equity, Diversity, and Inclusion Working Group (EDIWG), a cross Assessment Group (AG) committee.

The Digital Divide is often understood to be an inequity in availability of broadband internet access. Digital inequities create disparities in access to computing devices and technology as a whole, not just internet access. ${ }^{2}$ Computing devices and related communications technologies do not, by themselves, solve social and economic inequities, and can often exacerbate them. And new technologies do not always replace the old; it is often the case that we have old technologies meeting the needs for many people, while the new technologies make improvements in the lives of a select few. The key here is that it is always better for those institutions with the power to do so to meet people where they are rather than asking people to expend their limited resources trying to catch up to the institution's inexorable movement "forward."

We do not have all of the solutions in hand, rather we ask the Decadal Survey Committees, NASA, NSF, other funding agencies, and associated professional societies to consider our Recommendations within the context of the State of the Profession and other workforce issues that exist today and will exist in the future.

Recommendation \#1: The Planetary and Astrobiology Decadal Survey should consider the growing digital divide that impacts members of various communities and how this divide, if left unchecked, will seriously and negatively impact NASA's future workforce before the decade is out.

Recommendation \#2: NASA, NSF, and other funding agencies should acknowledge that their approach to mitigating the growing digital divide will impact issues discussed in the many DEIA white papers also presented to this Decadal Survey related to workforce issues. Agencies' particular approach to this issue will directly impact who gets to be a member of the Planetary Science and Astrobiology community for decades to come.

${ }^{1}$ The Exponential Growth of the Digital Divide

${ }^{2}$ The Digital Divide Among Students During COVID-19: Who Has Access? Who Doesn't? 
Recommendation \#3: The Decadal Survey Committee and NASA should make a concentrated effort to engage experts in the digital divide. These experts include educators, industry leaders in communications technologies, and other experts in fields as diverse as non-digital education, curriculum development, postal services, and informal and offline community engagement, systemic racism, and social psychology, among others. Related to this, The National Academies should consider incorporating concerns about the digital divide and its impact on future workforce for NASA, NSF, and other agencies in their existing education and DEIA focused virtual seminar series. ${ }^{3}$

Recommendation \#4: Professional societies (AGU, AAS, AAS/DPS, etc.) should consider how the digital divide may impact their DEIA initiatives and work with NASA, NSF, and other funding agencies to ensure that communities currently on the under-resourced side of the digital divide are not left behind in the coming decade.

COVID-19 Exposed major inequalities between households in lower, middle, and upper income brackets. A recent poll ${ }^{4}$ by the Pew Research Center found that: A) 36\% of children in lower income households were unable to complete their assigned homework because they did not have access to a computer at home; B) $40 \%$ of students had to travel out of the home to find a public Wi-Fi connection because their home does not have a reliable internet connection; and C) $43 \%$ of students in low-income households had to do their homework on a cellphone. The proportions of students in urban and rural settings were similar: Unable to complete homework A) $24 \%$ rural households compared with 33\% urban; Unreliable home internet B) 31\% rural and 30\% urban; Homework on a cellphone C) $33 \%$ rural and $36 \%$ urban. The digital divide negatively impacts students in both rural and urban households. Suburban households had significantly fewer issues. The survey also exposed another concern that is known to impact future performance of students: financial worries. The Pew report found that $28 \%$ of households with a high-speed broadband connection at home have been worrying about their ability to pay for the service over the months or perhaps years-long pandemic, and $30 \%$ of smartphone users (with data) worry at least some about paying their cell phone bill. The digital divide is especially problematic for Native Americans: at least $35 \%$ of Native American students have no access to broadband internet or cell phone service (so hotspots cannot work). Parts of these surveys are based on maps of broadband data access, but such maps generally do not provide a measure for the quality of the data connection. When learning is dependent on what one can afford, the divide between those who have and those who do not grows.

RAND Corporation surveyed K-12 schools about COVID-19 and the state of schools, the digital inequalities, and the impacts on students. ${ }^{5}$ Their report found that $75 \%$ of teachers think students on the under-resourced side of the digital divide face a serious obstacle to effective remote, digital learning. Parents and teachers agree that many of the potential problems might be

${ }^{3}$ Imagining the Future of Undergraduate STEM Education

${ }^{4} \underline{53 \% \text { of Americans Say the Internet Has Been Essential During the COVID-19 Outbreak }}$

${ }^{5} \underline{\text { COVID-19 and the State of K-12 Schools }}$ 
mitigated if schools lend mobile technology such as tablets and Wi-Fi hotspots to households. Of parents in low-income households, and single-parent households, $\sim 65 \%$ (and $\sim 75 \%$ of parents of students with severe or moderate disabilities) think they would find benefits in the lending of such technologies. . $^{5,6,7}$ One issue with lending technology is that fewer than $1 / 3$ of school districts surveyed had the capacity or plans to provide tablets, computers, or broadband hotspots to students, and even many rural districts that planned for this were unable to provide such technology to each student and instead have distributed such hotspots as well as they could. However, students (and working parents) were still required to travel long distances to access those hotspots. ${ }^{8}$

Costs increase the divide. A related problem is not just access to broadband internet services, but also the cost of data and the data cost of various educational solutions. For example, a one-on-one Zoom meeting for a single hour costs approximately 540 to $>800 \mathrm{MB}$ of data; a student required to be on video conferencing for 5-hours/day could easily accumulate up to $100 \mathrm{~GB} /$ month. If there are more students in a household, of course the data usage would be proportional to the number of students. This estimate does not include parents' or care-givers' data use if they are also working from home. The cost of broadband access varies wildly depending on many factors, including distance to the nearest networking hub. The average cost in the United States is about $\$ 70 /$ month ( $\$ 840 /$ year) for access to the very minimum plan that will allow video conferencing. Data overage fees can easily increase this cost by a multiple of 2 to $5 .{ }^{9}$ For many lower income families, these costs were never part of their budget. COVID-19 has suddenly and unexpectedly increased costs for some families.

Race impacts the divide. Several studies have shown that both access to the internet and owning a home computer vary by race. ${ }^{10}$ A Pew Research Report from its "Internet and American Life" Project in 2017 showed that $79 \%$ of white households were online compared to $70 \%$ of Black households and $63 \%$ of Hispanic households. ${ }^{11}$ They also looked at the demographics of those

\footnotetext{
${ }^{6}$ Parents' Survey Identifies Key Needs for New York Families Navigating New Reality

${ }^{7}$ The Disparities in Remote Learning Under Coronavirus (in Charts)

${ }^{8}$ For thousands of students without home internet access, remote learning is an extra challenge

${ }^{9}$ The Cost of Connectivity 2020

${ }^{10}$ Mapping The Digital Divide

${ }^{11}$ Who's Not Online And Why
} 
who (by necessity) use the internet outside of the home. Only $6 \%$ of White respondents needed to use the internet outside of the home as compared to $15 \%$ of Black respondents and $13 \%$ of Hispanic respondents. For these users, $50 \%$ of the reasons given for using the internet outside of the home related to cost and access. In 2017, the US Census Bureau published a graph to illustrate how race plays into the Digital Divide ${ }^{12}$, and it shows that the proportion of Black-alone

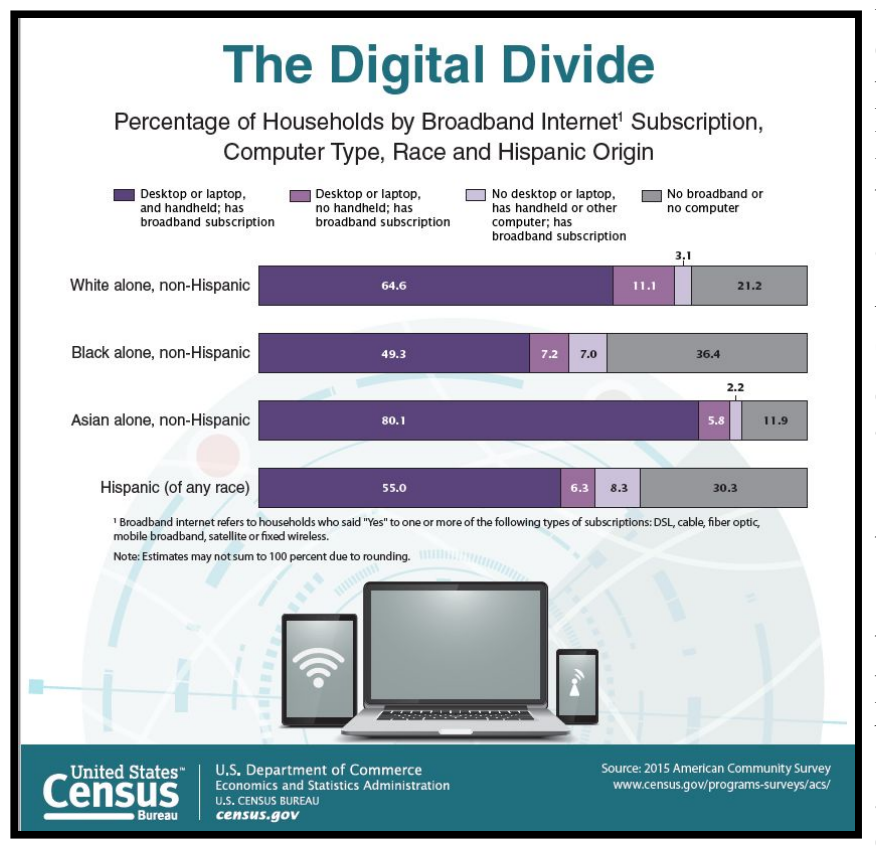
households that had no broadband connection and no home computer was $15 \%$ higher than White-only households. Hispanic-only households were 9\% higher than White-only households with no internet access or home computer. Native American, Alaskan Native, and Pacific Islander (NA/AN/PI) households are typically left off of these kinds of survey results because the "manifest destiny" genocide of the past several centuries has decreased their numbers to around $1 \%$ of the US population, which is smaller than the margins of error for these types of surveys. NA/AN/PI families are still here, of course, and are definitely impacted by the digital divide in similar and often greater proportions to other BIPOC communities. Indigenous and Native

American communities are also bearing a disproportionate fallout from the pandemic and climate change. ${ }^{13}$ We need to treat access to broadband and high-speed internet as an essential necessity, much like running water and electricity. Broadband internet has become critical to daily life at present, including remote learning, teaching, work, and many other essential needs such as telemedicine (physical and mental health). These needs are heightened when geographical and economic isolation are compounded by sheltering in place, and will not end with the pandemic as many employers and tech companies are planning on remote work being the new normal, increasing the economic gap between those with and without high-speed internet. Lack of access to adequate technologies, including the internet, threatens to hold American school children back a grade, and set its future workers back by years of missed learning and opportunities. This digital chasm will not be easily bridged in the near future without intentional and early mitigation at the federal and agency levels.

Disabilities can increase the divide. Because of the pandemic, K-12, undergraduate, and graduate schools are moving or have moved very swiftly to online, digital education models. The nature of the sudden change has meant that many issues have been left for later. Issues of Section 504 and 508 compliance, ${ }^{14}$ ADA compliance, student safety, and other considerations have often been left to the wayside in the rush to meet most students' needs, leaving people behind.

\footnotetext{
${ }^{12}$ The Digital Divide: By Internet, Computer, Race \& Hispanic Origin

${ }^{13}$ COVID-19 and Indigenous peoples | United Nations For Indigenous Peoples

${ }^{14}$ What is section 504 and how does it relate to Section $508 ?$
} 
Conversely, COVID-19 has also provided opportunities for some people with disabilities. In higher education spaces, there has been an increased interest in virtual/remote participation in field work (of geosciences field and lab courses surveyed, nearly $3 / 4$ transitioned to virtual instruction in the spring and summer. $)^{15}$ Virtualized instructional methods can be barriers when deployed haphazardly, but can provide increased accessibility for students when designed appropriately and when students have digital access. ${ }^{16}$ It will be important to not only find instances where the pandemic has increased problems with access, but also where solutions have been found that allow more people access. These solutions will help NASA, other agencies, and our community to model those successes and build greater resiliency to future disruptions.

The Divide impacts educators as well. Many teachers face similar challenges as their students. However, data costs for teachers can be much higher because they may be teaching more classes than students have to attend; teachers with poor access to broadband internet services may have trouble acquiring the digital materials needed to teach; teachers with low incomes may not be able to pay their internet service bills. In some districts, schools are going back to distributing paper packets for their students because neither the districts nor the students have the resources to go to online education. ${ }^{17}$ Around $47 \%$ of high-poverty rate districts in one survey do not have the financial resources to provide students with computing devices and internet access, and so a primary component of their education plans is physical distribution of learning materials. ${ }^{18}$

The American Institute of Geosciences has been collecting information on impacts, including statistics on impact of COVID-19 on fall budgets. ${ }^{19}$ At least $20 \%$ of respondents indicated that budget cuts involve salary reductions. In the survey results released (to June), non-tenured (and adjunct) faculty are twice as likely to be furloughed (10\% of respondents) than tenured faculty (5\%). Departments also reported furloughs/terminations for staff, which will impact the ability of those programs to support research facilities and/or may place additional administrative burdens on faculty. Also, "nearly three-quarters of students had fieldwork activities cancelled." While somewhat unique to the geosciences and Earth-related planetary sciences and astrobiology, a huge fraction of our student cohorts are either now behind in graduation requirements or will lack field/research experience when they apply for future positions. ${ }^{20}$

Summary of the Problem: COVID-19 has exposed and exacerbated the digital divide and digital inequalities especially for Black, Indigenous and People of Color (BIPOC); low income; urban; and rural families and students. This includes families and students with a wide variety of mental health issues and disabilities that can be negatively impacted by online educational models. It is well-understood that early access to STEM and technology in general has a positive impact on student performance, degree completion, and science and graduate school enrollment (11). This growing digital divide and its sudden increase caused by COVID-19 will have

${ }^{15}$ COVID-19 Impacts on Geoscience Academic Instructional Environments in Spring 2020

${ }^{16}$ Accessibility and Fieldwork in the Time of Coronavirus

${ }^{17}$ A 'Covid Slide' Could Widen the Digital Divide for Students

${ }^{18}$ National Survey on Public Education's Coronavirus Pandemic Response

${ }^{19}$ COVID-19 Impacts on Geoscience Academic Departments

${ }^{20}$ COVID-19 Impacts to Research Activities in Spring 2020 
negative impacts on NASA's workforce in 10 years or fewer, when these students are (or are not) graduating from high school or with undergraduate degrees and are making decisions about undergraduate and graduate school and majors.

Solutions to the Problem: Many currently-proposed solutions to the inequities of the digital divide and subsequent inequalities are aimed at addressing the short-term issues of the 2020-2021 academic year. ${ }^{21}$ These solutions are generally aimed at increasing students' access to technology and quick virtualization of curriculum, rather than considering other models of education. As mentioned earlier, some school districts have taken to distributing educational materials physically, which helps the students stay up-to-date with their education expectations, but does not mitigate the widening digital divide.

What can NASA, NSF, other agencies, and professional societies do to help with this problem? Many of the white papers submitted to this Survey that discuss workforce issues, including diversity, equity, inclusion, and accessibility have recommended solutions to some issues that are related to or directly connected to the digital divide, though many focus more on current workforce issues rather than on upcoming workforce issues. NASA's ROSES program regularly has multiple opportunities to propose to programs that address education and public engagement in various ways. Perhaps these programs could be modified or augmented to enable responses that bring expertise to bear on this problem.

Improving access to high-speed internet is a nontrivial problem; we have seen limited progress over the past two decades despite federal and private initiatives. Options offered through ground-based infrastructure tend to serve high-density population centers better than rural populations or low density areas. This is especially true where the terrain or large expanses of land may lead to spotty connections, or where local internet company monopolies lead to low-quality bandwidth. Solutions are not just ground-based but increasingly space-based, with the rapid increase of many thousands of privately funded satellites in low-Earth orbits. Large satellite constellations have the potential to bridge the digital divide, but it is not clear whether their promise of low-cost high-speed broadband internet will be achieved to the degree claimed by their owners. These constellations also come with new ethical concerns (c.f., 12). Such private actors partner closely with NASA and NSF. As a publicly funded agency, it is imperative that NASA, NSF, and other agencies hold these companies accountable to all of humanity and its pressing needs, especially when satellite constellations have already begun to have an enormous impact on scientific astronomy and dark skies. ${ }^{22}$

It's clear that the digital divide has not been solved, despite discussions about it for more than 20 years. It's also clear that the divide has worsened in recent years and especially in 2020 due to the global pandemic. The reality is that the authors of this paper are not the experts on how to close the digital divide so that the pool feeding Agencies' future workforce does not become restricted to those with access to resources. Instead of providing specific solutions to the problem in this white paper: We encourage NASA, NSF, and other funding agencies to quickly initiate a series of workshops and review boards, and to invite experts in education, digital

${ }^{21}$ Closing The K-12 Digital Divide In The Age Of Distance Learning

${ }^{22}$ Impact of Satellite Constellations on Optical Astronomy and Recommendations Toward Mitigations 
racism, equity, the digital divide, and information and communications technologies to begin to understand the problem and potential solutions. We have gathered some thoughts aimed at agencies and professional societies that could, perhaps, help kickstart the conversations:

1) Take quick action in the short term while investing in the long term. NASA and other funding agencies should, whenever possible, maintain some flexibility in its programs so that when crises such as COVID-19 and hurricanes impact members of its future community, Agencies can respond in helpful ways, but long-term investments in reducing the digital divide will be essential to meeting future workforce needs.

2) Help define digital (and possibly non-digital) STEM education long term approaches and applications. Invest in the future of the workforce through comprehensive engagement strategies that are specifically designed to reduce the digital inequalities.

3) Identify experts in the field of closing the digital divide and engage with them to help find ways to support these experts. This would include the private sector.

4) For NASA, NSF, and other funding agencies, both an equity and a justice lens should be applied before making major decisions. Agencies routinely consider their actions in light of legal constraints. It would be helpful to model a new Office of Equity and Justice after NASA's Office of the General Counsel. To accomplish this, NASA needs to engage and fund experts with experience in applying those lenses.

5) Use the current situation to look beyond the present to find ways to make meetings (and conferences and workshops) and information presented at meetings more widely accessible in the context of the digital divide; plan for follow-up assessments after meetings; determine the resource needs and availability of resources for meeting organizers to do such evaluations.

References: [1] Who is missing in Planetary Science?: A Demographics study of the planetary science workforce, Ed Rivera-Valentin, USRA/LPI; [2] Who is missing in Planetary Science?: Recommendations to increase the number of Black and Latinx scientists, Julie Rathbun, PSI; [3] Breaking Down Barriers: Accessibility in Planetary Science, Jen Piatek, CCSU; [4] Ensuring Inclusivity in the 2023 Planetary Science and Astrobiology Decadal Survey, Julie Rathbun, PSI; [5] Diversity in action: Solutions for a more diverse and inclusive decade of planetary science and astrobiology, Britney Schmidt, Georgia Institute of Technology; [6] LGBTQ+ in Planetary Science, Kathleen Vander Kaaden, Jacobs Technologies; [7] Professional development in the next decade: Supporting opportunities in all career paths and life events, Ryan Watkins, PSI; [8] The Power of the Solicitation: Exerting EDI Influence at the Institutional Level, Daniella Scalice, NASA ARC; [9] Addressing mental health in planetary science, Steve Vance, JPL; [10] Results of the 2020 Planetary Science Workforce Survey conducted by the American Astronomical Society (AAS) Division of Planetary Science (DPS), Amanda Hendrix; [11] STEM enrichment programs and graduate school matriculation: the role of science identity salience, Merolla, D.M., Serpe, R.T., Soc Psychol Educ 16, 575-597 (2013). https://doi.org/10.1007/s11218-013-9233-7; [12] The Low Earth Orbit Satellite Population and Impacts of the SpaceX Starlink Constellation, McDowell, J., ApJ 892, No. 2, (2020), 10.3847/2041-8213/ab8016; [13] Asteroid Resource Utilization: Ethical Concerns and Progress, Andy Rivkin, Johns Hopkins University Applied Physics Laboratory. 